\title{
Foreword for Special Issue
}

\begin{abstract}
Ningning Zhao
With the development of modern society and expansion of world population, the environmental pollution and chemical toxicity becomes a hot topic in public health researches. To evaluate the heavy metal content in ecosystems and food sources is of great interest, but at the same time it has high difficulties. The purpose of this special issue is to develop the remediation and management strategy and provide guidelines to avoid heavy metal exposures, and identify the data and knowledge gaps that need to be filled by future research.

In this special issue, authors from Sri Lanka have

elements in ten kinds of sea cucumbers by using sound method. This research provided novel and useful information on the general bioaccumulation pattern of heavy metals in marine organisms. In addition, it helps to develop general healthy diet guidelines.

As the guest editor of this issue, I would like to thank all the authors who submitted their manuscripts to this Special Issue. I would also like to thank all the reviewers who evaluated the merit of the manuscripts and made helpful suggestions to the authors. Without their contributions, this Special Issue will not be present.
\end{abstract} thoroughly measured the levels of a variety of trace 